\title{
Entrelacs
}

Cinéma et audiovisuel

$17 \mid 2020$

$N^{\circ} 17$ / Enjeux Audiovisuels du cinéma 360

\section{Construction de l'espace scénographique et ubiquité}

Olivia Dorado

\section{(2) OpenEdition \\ Journals}

Electronic version

URL: http://journals.openedition.org/entrelacs/5917

DOI: 10.4000/entrelacs.5917

ISSN: 2261-5482

Publisher

Éditions Téraèdre

Electronic reference

Olivia Dorado, "Construction de l'espace scénographique et ubiquité », Entrelacs [Online], 17 | 2020

Online since 01 July 2020, connection on 03 July 2020. URL : http://journals.openedition.org/

entrelacs/5917; DOI : https://doi.org/10.4000/entrelacs.5917

This text was automatically generated on 3 July 2020 .

Tous droits réservés 


\title{
Construction de l'espace scénographique et ubiquité
}

\author{
Olivia Dorado
}

1 Le cinéma à $360^{\circ}$ promet en quelque sorte une expérience exploratoire et modifie la position spectatorielle, ainsi que la nature-même du concept d'espace. Sa place et son interprétation au sein de l'objet filmique s'en trouvent radicalement modifiées, ne serait-ce que par la mutation de l'interaction existant entre le décor et le spectateur, tant en termes de compréhension et d'appréhension qu'en termes d'appropriation. L'extension du domaine spatial étant issue d'une volonté de réalisation atypique, il en découle une évolution des intentions narratives et esthétiques propres à bouleverser la conception du décor actuelle, tant sur le point technique que sur les fondements intrinsèques.

2 Il est nécessaire aujourd'hui de se pencher plus particulièrement sur cette représentation de l'espace dans le cinéma à $360^{\circ}$ qui amène à une redéfinition totale du concept spatial au sein du dispositif cinématographique.

\section{1 - La représentation de l'espace au sein du cinéma traditionnel}

3 Quand il est question de représentation de l'espace au cinéma, il ne s'agit pas d'une référence exclusive au décor. On peut également entendre une notion plus abstraite, une dimension structurelle.

4 André Gardiès, Professeur d'Université en études cinématographiques et écrivain, distingue quatre types d'espace liés au cinéma ${ }^{1}$ :

L'espace cinématographique », qui est celui dans lequel se trouve "immergé ou exposé le spectateur $»^{2}$ et qui lui permet de recevoir le film. On y accède à partir du moment où l'on choisit un siège dans la salle de projection. Cet espace extrait l'individu de son quotidien en le plaçant dans une position d'acceptation d'un dispositif, d'une œuvre. Ainsi, la localisation du spectateur dans le dispositif de 
projection constitue en soi un espace de réception et de perception à part entière, capable d'influer sur les autres espaces cinématographiques.

"l'espace diégétique», qui est l'espace que construit le film "comme réalité indépendante du récit $»^{2}$. Gardiès distingue ici l'espace et le lieu, l'espace diégétique constituant ici une construction entre les lieux apparaissant dans le film et l'appel au savoir du spectateur en ce qui concerne sa perception d'un "lieu commun ", d'une histoire commune. L'espace diégétique est intrinsèquement dépendant $d u$ milieu socio-culturel du spectateur, mais il est tout de même possible de faire appel à des références spatiales appartenant à un savoir partagé.

«l'espace narratif », qui renvoie aux lieux, à la spatialité dans lesquels évoluent les personnages au sein de l'objet filmique. Cet espace est perçu comme un partenaire de l'acteur au sein de la narration dans le sens où il communique, au même titre que l'acteur, une action ou une représentation qui vont alimenter la construction narrative $\mathrm{du}$ film. L'espace devient un personnage muet entrant en interaction directe ou indirecte avec les acteurs.

«l'espace du spectateur », qui est en quelque sorte celui du lien qui va être créé entre la narration et le spectateur, un espace déterminé par le rapport du spectateur à la stratégie de communication employée ou à l'intention de réalisation. Cet espace reste inévitablement lié au caractère unique de cette relation privilégiée et demeure contraint par la perception propre à chaque spectateur de la nature des moyens filmiques employés (dans et autour du film).

Dans la pratique, au cinéma dit traditionnel, ces espaces sont distincts même si des interactions existent.

6 Le décor cinématographique trouve son application concrète au sein de l'espace narratif et de l'espace diégétique. À ce titre, on peut considérer que l'espace narratif sera au service de l'espace diégétique, et inversement, l'espace narratif permettant une sorte de cadre spatial à l'action.

7 Par souci de différenciation, nous ferons le choix de nommer le décor cinématographique construit "espace scénographique». Celui-ci, concrètement façonné par la main des techniciens se situe, par sa nature, en opposition aux décors naturels bien qu'il soit situé sur l'espace narratif et l'espace diégétique au même titre.

Dans le cinéma traditionnel, nous pouvons observer une fragmentation de cet espace scénographique. Premièrement car cet espace est limité dans sa perception visible par le cadre mais également parce qu'il peut être construit de manière fragmentée au sens propre. Il est une évidence que le cinéma propose une fragmentation temporelle et spatiale, mais ce qui va déterminer la cohérence ou le sens de l'objet filmique va notamment se situer dans la conception de cette fragmentation de l'espace scénographique par le chef décorateur et le chef opérateur. Par ailleurs, la perception de cette cohérence spatiale par le spectateur est également tributaire de son propre champ d'appréhension, combiné à des savoirs d'ordres personnel et collectif acquis au cours de son existence. Le champ d'appréhension désigne l'ensemble des éléments pouvant être sensoriellement considérés, retenus et compris après une brève exposition. D'une personne à l'autre, le nombre d'éléments pouvant être perçus simultanément varie, tout comme la correspondance appréhension-compréhension de chacun de ces éléments est influencée par les références personnelles et collectives qui y sont associées. ${ }^{3}$ 


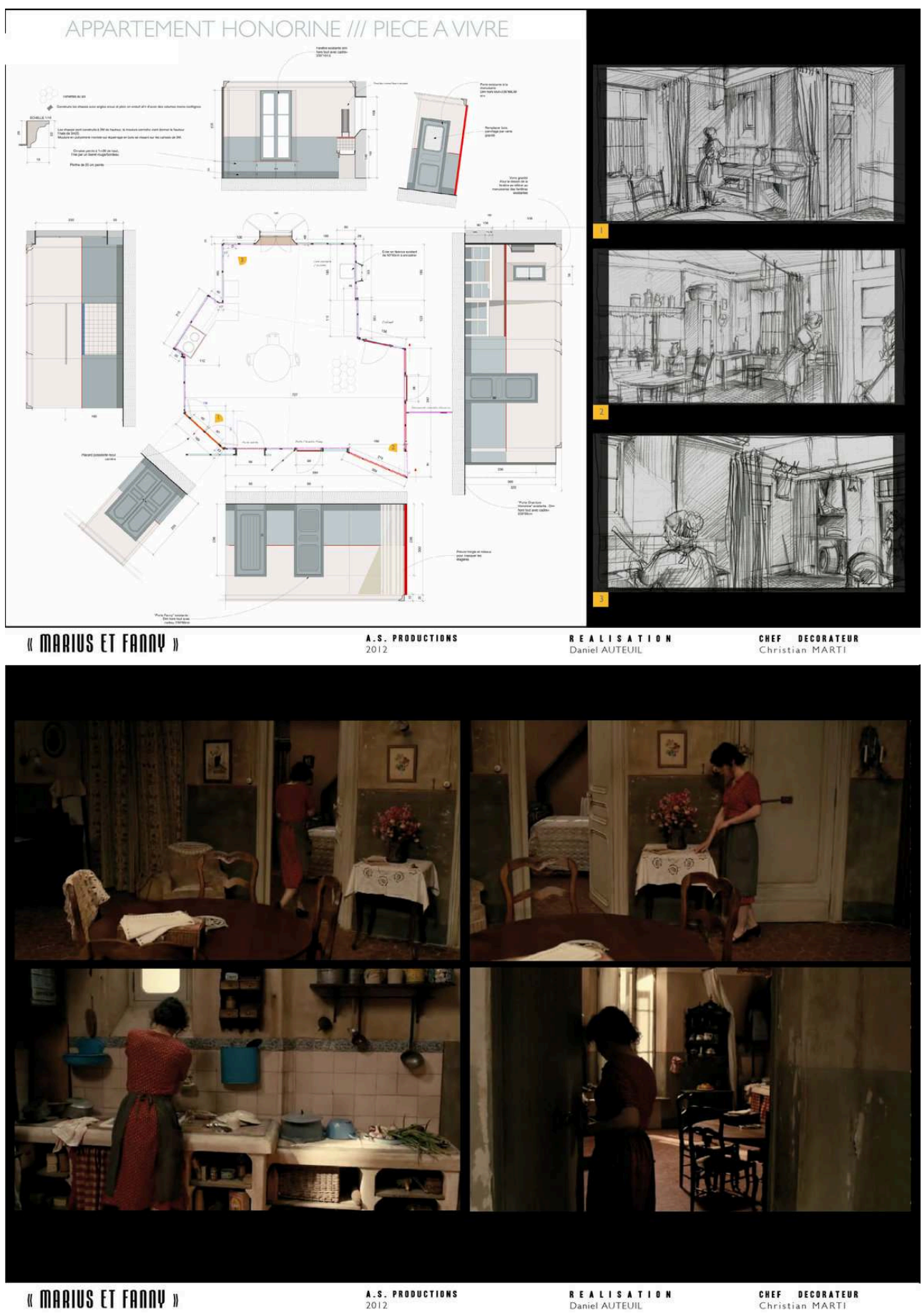

Illustration 1 : Plan, croquis et photogrammes de Gladys GAROT, 1 ère assistante dessin pour les décors de Marius et Fanny (Chef décorateur : Christian MARTI). Daniel Auteuil (réalisateur). 2012. A. S. Productions.

Que ce soit au sens propre ou au sens figuré, un décor cinématographique est par définition " éclaté », puisqu'il ne sera réellement visible qu'à travers les limites du cadre déterminé par l'opérateur ou tout simplement construit partiellement et avec des éléments distincts. Mais ce décor, cet espace de jeu, trouvera par la suite sa complétude dans les raccords visibles et invisibles mis en place. Soit par le montage, soit par une action, un mouvement de caméra, soit par des éléments, des accessoires qui vont se retrouver d'un plan à l'autre. Mais cette unité peut aussi se construire par le biais d'un 
langage verbal ou sonore. Jean-Luc Antonucci, parlait ainsi d'un «assemblage des différents fragments visuels collectés associés à des sons particuliers qui va produire le sens voulu par le réalisateur $»^{4}$. Cette intention, ce « sens voulu par le réalisateur », sera obtenu à force de gestion de contraintes et d'aléas aussi bien que d'apports techniques et esthétiques issus des différentes contributions de l'équipe.

Cependant, au-delà des questions logistiques et techniques, cette fragmentation visuelle délibérée et déterminée de l'espace scénographique devient également une technique narrative et un choix identitaire propres au film. Qu'il s'agisse de décors naturels ou de décors construits au cinéma, l'espace du décor visible est tributaire des dynamiques spatiales inhérentes au cadre et au montage qui vont servir la dynamique narrative.

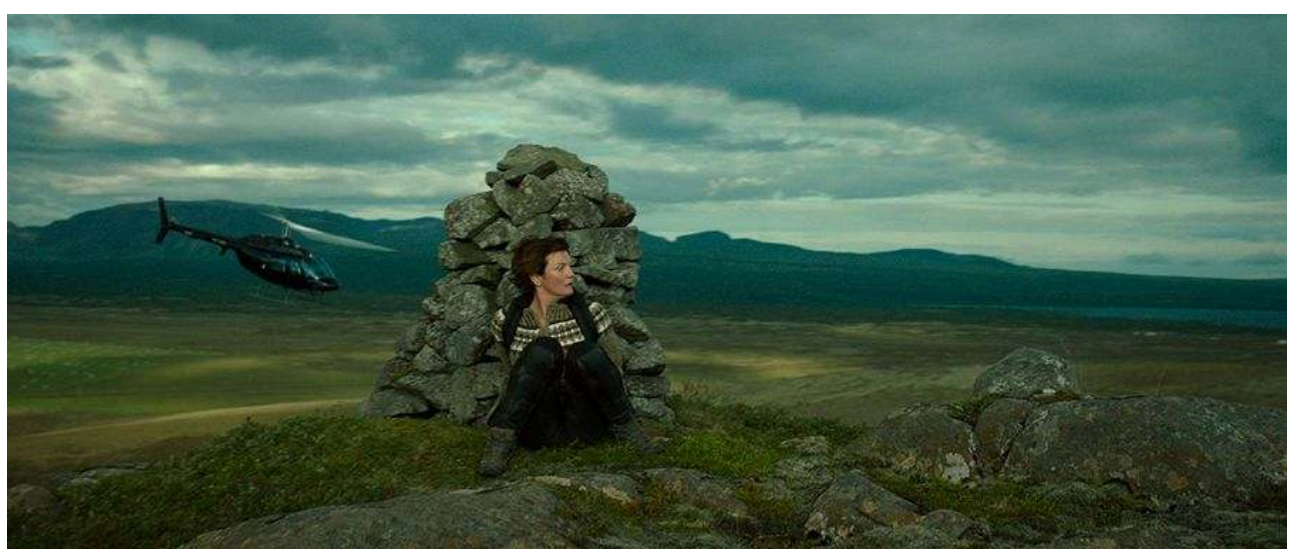

Illustration2 : Photogramme du film Woman at war. Benedikt Erlingsson (réalisateur). 2018. Sena, Jour2fête. [DVD]

Lors de la constitution de cet espace scénographique, la valeur et les modalités de sa monstration vont qualifier de manière déterminante une partie du scénario et des personnages. Il y a donc un choix évident qui est fait entre le visible et l'invisible, chacun donnant sens à la genèse mise en place. Il y a une volonté assumée de définir un espace grâce à une combinaison de champs et de hors-champs, que je distingue en visible, invisible et suggéré. La différence entre "l'invisible » et le "suggéré » se jouant sur le fait que le fragment de décor «invisible» est volontairement annihilé dans la conception de l'espace perçu contrairement à l'espace suggéré, que l'on ne voit pas mais que l'on imagine (donnant lieu à la constitution d'un fragment de l'espace diégétique). 

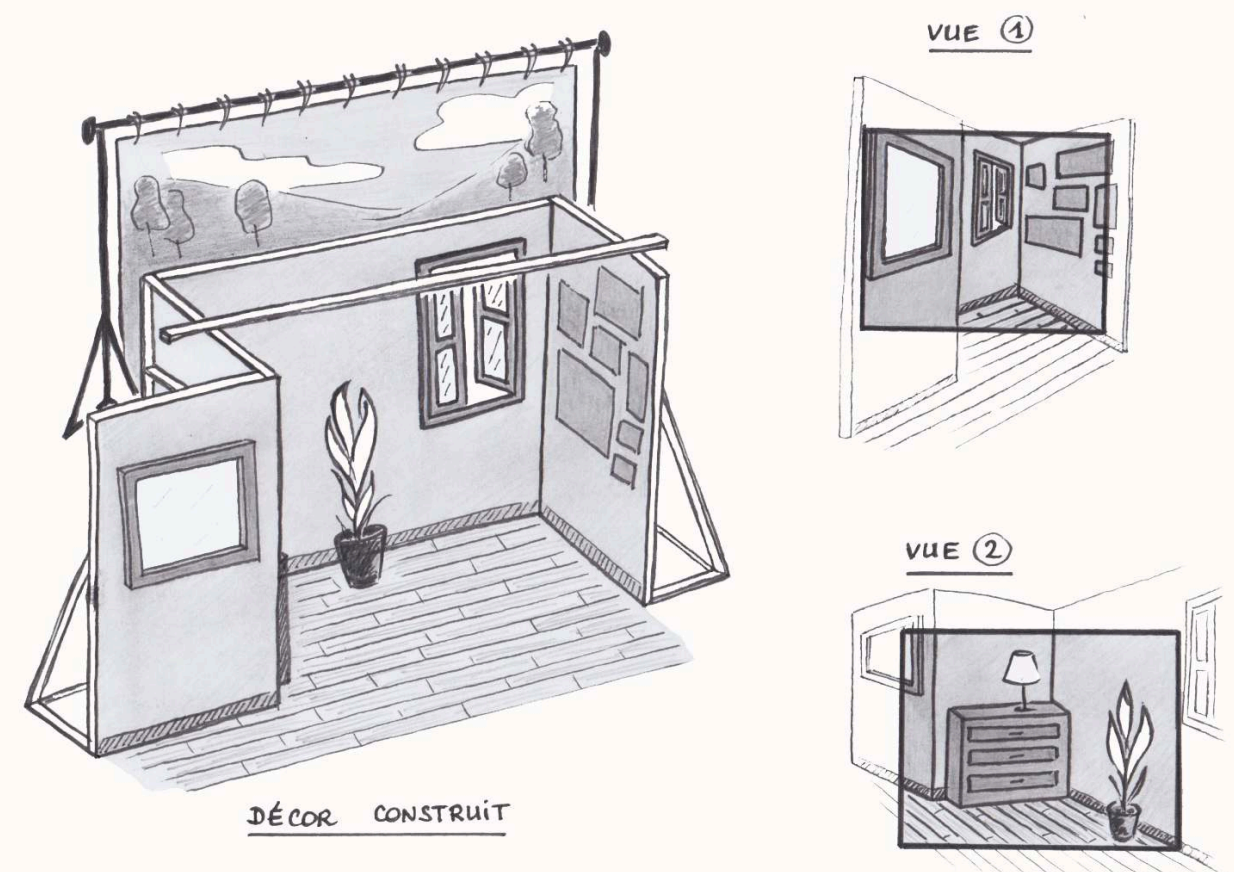

Illustration 3 : Plan, croquis d'un décor construit et de vues possibles de zones visibles, invisibles, suggérées. Olivia Dorado

Au cinéma, si nous nous plaçons d'un point de vue spectatoriel, le décor est perçu. Il est interprété. Comme entier, comme un «tout ", grâce à la succession et l'agencement de fragments divulgués ou non de ce décor. L'espace scénographique de l'espace narratif est utilisé pour construire chez le spectateur la notion d'un espace diégétique imaginé, celui-ci étant construit autour d'une structure elliptique. Pour mieux se rendre compte de ce phénomène perceptif, il est pertinent de faire l'analogie avec la compréhension fondamentale que nous avons d'une image en mouvement. Cette illusion perceptive n'est pas due uniquement au phénomène de persistance rétinienne mais également au fait que les mécanismes cérébraux comblent l'absence de transition entre les images de la manière la plus logique possible pour nous donner une impression de mouvement. Ce principe s'appuie sur l'association des phénomènes Phi et Beta ${ }^{5}$ (le premier traitant de la logique d'une proximité temporelle entre les images et le second impliquant une logique de continuité entre ces images). De la sorte, la sensation visuelle d'un mouvement perçu à partir d'images fixes (qui se succèdent et sont projetées à une vitesse de succession raisonnable pour un mouvement continu) est issue de l'appropriation de ces ellipses par le champ d'appréhension du spectateur. Ainsi, en dépassant l'aspect strictement anatomique pour y adjoindre des approches psycho et neurophysiologiques, le spectateur n'est plus considéré comme récepteur passif mais au contraire comme récepteur actif. Sur ce même principe, nous pouvons arguer que, face à un espace diégétique proposé (celui-ci étant fondamentalement incomplet), le spectateur va effecteur une réception active en réalisant une construction mentale vraisemblable de cet espace. Cette reconstitution spatiale va être possible en comblant intellectuellement les manques (l'invisible) grâce à une logique de continuité entre les espaces visibles et/ou suggérés, tout en prenant en compte une logique de proximité temporelle entre ces espaces visibles et/ou suggérés tout au long du film. 
13 Le dispositif cinématographique classique induit donc chez le spectateur l'illusion de la maîtrise du concept de cet espace. Il lui permet de le comprendre, de le reconstituer, en faisant appel à une logique issue d'un savoir collectif combiné à son champ d'appréhension, tout en le soustrayant pourtant à une perception simultanée des différents lieux de ces espaces narratifs et diégétiques.

Cette perception simultanée est possible, dans une certaine mesure, au sein du cinéma à $360^{\circ}$. Il est à noter que si le hors-champ peut se définir par une existence hors du visible de la caméra, celui-ci étant délimité par les bords cadre, il est important de considérer deux types particuliers de hors-champ : celui se situant derrière la caméra et celui situé à l'intérieur même du cadre. Le hors-champ localisé derrière la caméra, considérée comme point de vue omniscient pour l'objet filmique, tend à disparaître dans un dispositif de prise de vue à $360^{\circ}$. Cette altération de l'omniscience narrative du point de vue de la caméra implique alors une redéfinition du schéma narratif. Par opposition, le hors-champ présent à l'intérieur de l'image (pouvant être constitué par un élément ou un personnage dissimulé par un élément de décor situé dans le même espace de jeu) ne pourra se dévoiler qu'en fonction de la volonté du réalisateur.

\section{2 - L'illusion d'ubiquité suggérée par le cinéma à 360}

À partir de ce constat, comment se déroule cette sensation spectatorielle d'immersion, d'ubiquité au sein du dispositif à $360^{\circ}$, alors même que la notion elliptique ne peut plus être pratiquée ? Comment se redéfinit l' « espace spectatoriel » et dans quelle mesure la posture même d'appréhension diégétique est impactée?

16 L'ubiquité est le fait d'être présent partout à la fois ou en plusieurs lieux en même temps. Il s'agit, dans le cas qui nous concerne, de la capacité d'être présent en tout lieu de cet espace scénographique.

17 Le cinéma à $360^{\circ}$ offre la possibilité au spectateur de s'approprier l'intégralité apparente d'un décor, d'avoir accès visuellement à la totalité d'un espace narratif. L'œil du spectateur a donc la capacité d'aller en plusieurs lieux de ce décor et donc de dépasser le champ d'un cadre. Nous allons donc distinguer le cinéma à $360^{\circ}$ et le cinéma réellement immersif qui permet au spectateur de se déplacer au sein de ce décor grâce à un dispositif technique spécifique.

18 Dans ce dispositif à $360^{\circ}$, la sensation première du spectateur va être d'être placé au cœur du décor. L'illusion d'ubiquité qui peut en découler vient essentiellement du fait que l'œil sera sollicité à $360^{\circ}$, celui-ci aura la possibilité de voir l'intégralité d'un espace scénographique et non plus des fragments assemblés via un montage. L'œil deviendra caméra, et c'est ce mouvement rotatif de la tête qui donnera un sens à l'espace offert et qui par extension permettra de construire une partie de la dynamique narrative, à l'image de l'appareil de captation cinématographique. Cependant, ce dispositif implique une simultanéité des différents types d'espaces dégagés par André Gardiès, plaçant l'espace du spectateur en concomitance avec les espaces cinématographique et narratif.

Si l'on part du principe que l'ubiquité implique la possibilité d'une présence en différents lieux à la fois, il convient de déterminer que le cinéma à $360^{\circ}$, que l'on peut dire immersif d'une certaine façon, propose davantage un système panoptique (i.e. une 
organisation architecturale, spatiale permettant d'observer l'ensemble de l'intérieur de cet espace sans pour autant être vu).

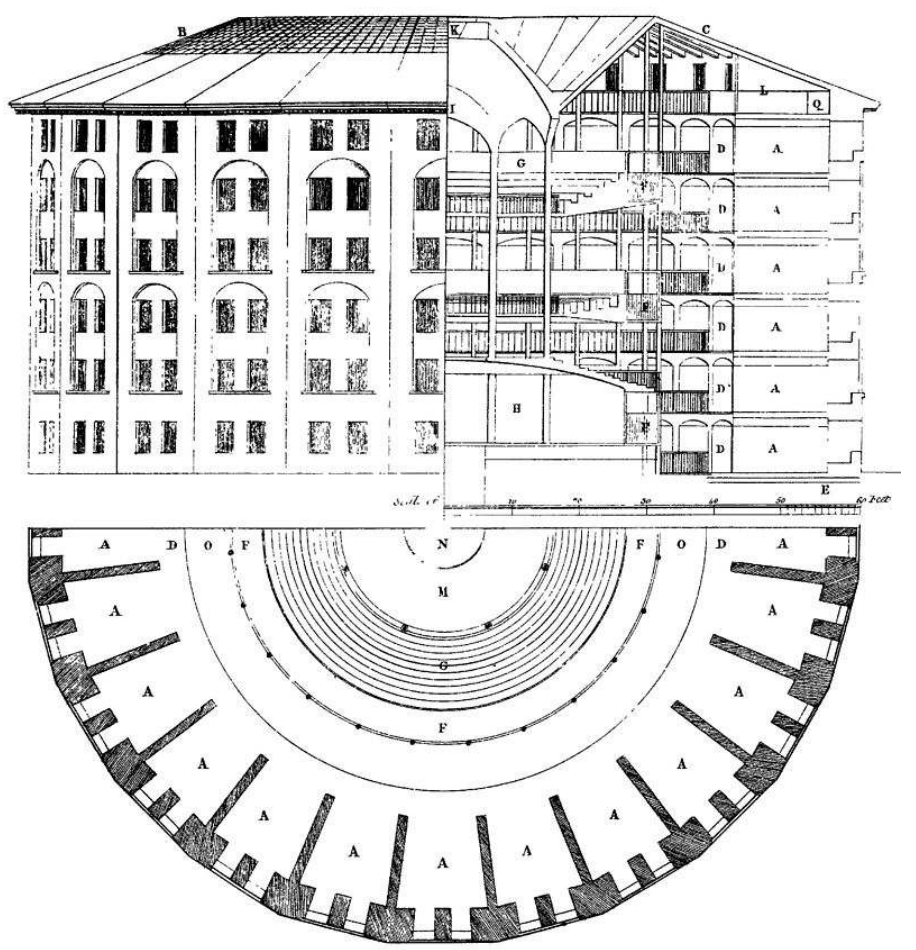

ILLUSTRATION 4 : PLAN DU PANOPTICON. THE WORKS OF JEREMY BENTHAM VOL. IV, 172-3

À noter que ce principe architectural a vu le jour fin XVIIIe siècle, avec pour objectif l'accompagnement d'une réforme du système carcéral. Créé par le philosophe utilitariste Jeremy Bentham, le système panoptique permettait l'observation permanente et jouait tout particulièrement sur l'antagonisme voir/être vu. Au-delà même de la fonctionnalité de cette structure architecturale en termes financiers et logistiques, il en découlera une notion plus philosophique amenant à en déduire que cette possibilité de donner le sentiment de pouvoir tout voir sans être vu (et à l'inverse donner l'impression d'être vu en permanence) entraîne un « assujettissement réel » axé sur le contrôle social selon Michel Foucault ${ }^{6}$. Ce concept structurel prend donc immédiatement une dimension sociale forte, impliquant un rapport hiérarchique lié au contrôle, à l'ascendant et à l'appropriation. Selon Gilles Deleuze, « la formule abstraite du Panoptisme n'est plus « voir sans être vu», mais « imposer une conduite quelconque à une multiplicité humaine quelconque ${ }^{7}$ ».

Évidemment, l'objectif et les fondements du cinéma à $360^{\circ}$ ne sont pas à associer à ceux de Bentham, d'autant que ce dispositif cinématographique ne permet pas d'être vu puisqu'il n'y a actuellement pas d'interaction possible entre les différents spectateurs participants à l'expérience. Par opposition aux jeux vidéo, au sein desquels la possibilité d'être visible par les autres participants est réalisable par l'intermédiaire d'un avatar (lui-même n'étant qu'une représentation d'un autre joueur).

Le cinéma à $360^{\circ}$ provoque une synchronie de l'espace cinématographique, de l'espace narratif et de l'espace du spectateur puisque désormais le décor scénographique à proprement parler est visible de manière "totale», englobant le spectateur dans 
l'espace narratif. Mais finalement ces espaces se confondent avec l'espace cinématographique puisqu'ils sont également la source permettant de voir le film.

Il convient cependant d'acter que cet espace reste défini, limité, comme dans une boîte puisqu'il ne sera possible au spectateur de déplacer son regard uniquement dans l'espace proposé par le réalisateur. Cet espace n'est pas infini mais en donne pourtant l'impression, dans le sens où le spectateur a le sentiment d'être libre de se déplacer à l'infini où il le souhaite.

Il n'existe donc pas d'ubiquité réelle au point de vue de l'image, du décor. Mais davantage de panoptisme puisqu'il s'agit finalement d'un dispositif permettant de tout voir sans être vu. Ou plutôt de donner l'illusion de tout voir depuis un point de vue unique.

\section{3 - Redéfinition de la narration spatiale au sein de ce dispositif}

Le spectateur se trouve donc en immersion au sein d'une "boîte ", à l'image du cadre cinématographique traditionnel. Cette organisation spatiale qui l'entoure ayant les limites conférées par l'illusion de la mise à disposition d'un décor total.

J'entends par là qu'il y a un espace supplémentaire qui est omis dans les 4 types d'espaces au cinéma mentionnés par André Gardiès 8: l'espace technique. Un espace constitué pour et par les différentes équipes techniques lors des prises de vue, capable de dissimuler matériels et techniciens.

Lorsque l'on fragmente un décor en plusieurs éléments, il y a la plupart du temps une justification logistique et financière (gain de place, moindre coût...) mais il y a également un aspect technique qui est systématiquement pris en compte: où va se placer l'ingénieur du son, le chef opérateur, où va-t-on installer la machinerie, les éclairages, comment va-t-on traiter les brillances et les reflets, etc.

Si l'on considère que dans le cinéma à $360^{\circ}$ le spectateur a accès visuellement à la couche superficielle du décor, la $1^{\text {ère }}$ face visible, ce dispositif a la particularité de ne laisser apparaitre aucun stigmate de sa conception alors même qu'il est explorable de bout en bout. Quid des câbles, pieds, mallettes, outils et appareillages? Comment occulter les techniciens agissant au moment de la prise de vue? La mise en place de ce nouveau dispositif implique l'élaboration de stratagèmes de dissimulation alternatifs ou l'utilisation d'une post-production visant à gommer les éléments et exécutants.

30 L'utilisation du décor "éclaté ", le principe de défragmentation du décor ne peut s'appliquer au cinéma à $360^{\circ}$, tout du moins s'il y a possibilité pour le spectateur de naviguer au sein du décor. Il est donc nécessaire de proposer un dispositif de décor qui «brise » la méthodologie usuelle puisqu'il nécessite un décor ne cachant aucun de ses éléments. Y compris l'équipe technique.

31 Cette question finalement nous amène à une autre : peut-on considérer que le décor et sa représentation telle que l'on a coutume de les envisager trouvent une nouvelle forme à travers le cinéma immersif ? Techniquement, comment le chef décorateur doitil procéder pour construire un décor visible dans ses moindres recoins?

À la différence d'un Escape Game ou d'un spectacle / théâtre immersif, le cinéma implique de nombreux techniciens œuvrant sur l'espace cinématographique même. Un 
décor cinématographique immersif implique donc l'effacement de cette réalité pour en créer une nouvelle.

Car s'il existe en effet des tournages où les décors sont construits dans leur totalité, en respectant une structure naturelle que l'on peut envisager comme étant à $360^{\circ}$ puisqu'il est possible pour l'acteur d'y naviguer comme dans un lieu réel, il faut prendre en compte que ces dispositifs sont dédiés à des films tournés en "coupures». Le spectateur n'a pas la liberté d'explorer et éprouver physiquement ce décor, il observe des acteurs qui s'y trouvent et il assiste à une séquence montée. Par opposition, le cinéma immersif donne la possibilité au spectateur de "vivre » au cœur de ce décor, avec une sensation d'immersion dans une réalité filmée, alors qu'il est évident que trucage il y a. Sans quoi le spectateur se trouverait en permanence confronté à l'équipe technique en place.

\section{CONCLUSION}

Face à ce nouveau dispositif filmique, le technicien décorateur doit abandonner le langage cinématographique et la dialectique architecturale conventionnels pour adopter une spatialisation du décor au-delà du cadre visible. Il reste évident que l'on ne peut pas pour autant affirmer que le hors-champ disparaît avec ce dispositif panoptique, considérant qu'il reste malgré tout une partie du décor invisible (celle audelà des murs, au-delà de l'horizon compte-tenu que le spectateur n'aura la possibilité d'explorer que le décor filmé et donc mis à disposition). Malgré tout, il n'en reste pas moins que le cinéma à $360^{\circ}$ redéfinit les notions d'espace au cinéma et amène à une réinvention de la conception scénographique de l'espace dans la manière de construire un décor, tout comme dans l'intention de réalisation en son fondement étant donné que le réalisateur devra prendre en compte les contraintes techniques et visuelles qui s'imposent.

Le cinéma immersif amène donc à se demander si l'on peut considérer que le "partout» est un "nulle part» matérialisé et dans quelle mesure l'immanence de l'espace scénographique peut être acceptée.

\section{BIBLIOGRAPHY}

\section{Bibliographie}

ANDERSON, Joseph et FISHER, Barbara. " The Myth of Persistance of Vision ». Journal of the University Association XXX: 4 (Fall 1978): 3-8, University of Wisconsin-Madison. URL : https:// faculty.uca.edu/wsmeador/ccsmi/ccsmi/classicwork/Myth1.htm [consulté le 21/04/20] ANTONUCCI, Jean-Luc. « Perspective et constructions ». Entrelacs [En ligne], 13 | 2017, mis en ligne le 18 mai 2017, consulté le 14 janvier 2020. URL : http://journals.openedition.org/entrelacs/ 2023 ; DOI : 10.4000/entrelacs.2023 [consulté le 06/02/20]

AUMONT, Jacques. L'Image. Paris, éditions Nathan, 1990. 254 p. 
DELEUZE, Gilles. Foucault. Paris, Editions de Minuit, 1986, 144 p.

FOUCAULT, Michel. Surveiller et punir. Naissance de la prison. Paris, Gallimard, 1975, 313 p.

GARDIÈS, André. L'Espace au cinéma. Paris, Méridiens Klincksieck, 1993, 222 p.

LÉVY, Jacques. « De l'Espace au cinéma ». Annales de géographie, n 694, 2013/6, Armand Colin, pp. $689-711$

RAMSEYER, Leo. « Louis Seguin et la question du hors-champ : une cartographie de l'espace du cinéma ». Décadrages [en ligne], n¹-2 « Le hors-champ », 2003, pp. 110-120. URL : https:// journals.openedition.org/decadrages/590 [consulté le 06/02/20]

SIMONDON, Gilbert. Simondon Gilbert, Cours sur la perception (1964-1965). Paris, Presses Universitaires de France, « Hors collection », 2013, 448 p., URL : https://www-cairninfo.gorgone.univ-toulouse.fr/cours-sur-la-perception-1964-1965--9782130625292.htm [consulté le $26 / 05 / 2020$ ]

WERTHEIMER, Max. « Experimentelle Studien über das Sehen von Bewegung ». Leipzig, Barth, 1912. $105 \mathrm{p}$.

\section{NOTES}

1. André GARDIÈS, L'Espace au cinéma, Paris, Méridiens Klincksieck, 1993, ces notions font l'objet du livre: 1ère partie: l'espace cinématographique; 2ème partie: l'espace diégétique; 3ème partie : l'espace narratif : 4ème partie : l'espace du spectateur.

2. Jacques LÉVY, « De l'Espace au cinéma », Annales de géographie, n 694, 2013/6, p. 690

3. Gilbert SIMONDON, Cours sur la perception (1964-1965), Paris, Presses Universitaires de France, 2013, pp. 285-319. « En conclusion, on peut dire que la perception de l'espace et du relief des objets met en jeu une pluralité de facteurs, dont certains sont, comme le langage, matière à conventions culturelles et s'intègrent à la perception humaine du milieu de vie. ", p. 319.

4. Jean-Luc ANTONUCCI, «Perspectives et constructions», Entrelacs [en ligne], n 13 «Espace, perspective et fragmentation », mai 2017, p. 6, URL : https://journals.openedition.org/entrelacs/ 2023 [consulté le 06/02/20]

5. Max WERTHEIMER, Experimentelle Studien über das Sehen von Bewegung («Etudes expérimentales sur la perception du mouvement »), Leipzig, Barth, 1912. L'ouvrage est le premier essai scientifique faisant état des expériences déterminant les effets Phi et Beta.

6. Michel FOUCAULT, Surveiller et punir. Naissance de la prison, Paris, Gallimard, 1975, p. 204.

7. Gilles DELEUZE, Foucault, Paris, Editions de Minuit, 1986, p. 41

8. André GARDIÈS, L'Espace au cinéma, op. cit.

\section{ABSTRACTS}

Abstract 
The complete view achieved thanks to an immersive display raises the question of cinema settings. If the spectator can move inside the scenographic space, it involves a mutation of its conception, apperception and intention. From its original representation to its immersive interpretation, the strengthening of the ubiquity illusion specific to this panoptic device translates into a technic space alteration.

Résumé

Le point de vue total obtenu avec un dispositif de cinéma immersif pose la question de la place du décor en son sein. L'espace scénographique dans lequel peut évoluer le spectateur voit sa nature et sa construction se transformer, impliquant une mutation de sa conception, de son appréhension et de son intention. De sa représentation originelle à son interprétation immersive, le renforcement d'une illusion d'ubiquité propre à ce dispositif panoptique se traduit par l'altération de l'espace technique envisagé.

\section{AUTHOR}

\section{OLIVIA DORADO}

Doctorante à l'Université de Toulouse Jean-Jaurès au sein de l'équipe de recherche du LARASEPPIA. Ancienne cheffe décoratrice dans le cinéma et le spectacle vivant, ses recherches tout comme son travail sont particulièrement attachés à l'aspect sensible de la création par les effets optiques et mécaniques. Son sujet de thèse porte sur la revalorisation des techniques d'effets spéciaux à la prise de vue par le biais de l'hybridation. En parallèle à ses travaux de recherche, elle est chargée de cours à l'ENSAV sur les exercices se déroulant au plateau décor. 